\title{
Prevalence of antinuclear antibody in patients with multiple sclerosis: a case- control study
}

\author{
Hind Alnajashi ${ }^{1 *}$ and Foziah Alshamrani ${ }^{2}$
}

\begin{abstract}
Background: Antinuclear antibody (ANA) is a common test for excluding alternative diagnoses. However, the significance of ANA testing in patients with multiple sclerosis (MS) remains unclear.

Objectives: To compare the prevalence of positive ANA antibody and its titer between patients with MS (cases) and non-MS patients who attended neurology clinics (control) in Saudi Arabia.

Methods: A case-control review of ANA results for all patients who attended a neurology MS clinic. We compared a convenience sample of patients with MS with individuals with general neurology problems and no known autoimmune diseases.

Results: There were 115 and 103 participants in the MS and control group, respectively. The mean age in the MS and control group was $33.76 \pm 8.96$ years and $34.95 \pm 8.56$ years, respectively. In the MS group, $25.22 \%, 60 \%$, $11.30 \%$, and $3.48 \%$ were negative, mildly positive, moderately positive, and strongly positive for ANA, respectively. In the control group, there were $34.95 \%, 54.37 \%$, and $10.68 \%$ were negative, mild positive, and moderate positive, respectively. There were numerically, but not significantly, more positive cases in the MS group (74.78\%) than in the control group (65.05\%) $(p=.117)$.

Conclusion: ANA testing in routine MS screening for excluding alternative diagnoses should be discouraged unless there is a remarkable history or clinical examination finding. Mild positive ANA is common among patients with MS and does not significantly differ from the general population.
\end{abstract}

Keywords: Multiple sclerosis, Autoantibody in neurology, Antinuclear antibody

\section{Introduction}

Multiple sclerosis (MS) is one of the most common central nervous system autoimmune diseases. The diagnosis is based on the 2017 McDonald criteria, which allows for early diagnosis; however, this should be made only if there is no better explanation for the existing symptoms [1]. The term "no better explanation" is vague since there is no clear diagnostic test for excluding alternative diagnoses. However, abnormal lab results should serve

\footnotetext{
* Correspondence: Halnajashi@kau.edu.sa

${ }^{1}$ Neurology Division ,Department of internal Medicine, Faculty of Medicine, King Abdulaziz University, Jeddah, Saudi Arabia

Full list of author information is available at the end of the article
}

as "a red flag." To this effect, necessary laboratory tests include cell blood count, liver and kidney function test, thyroid function, ANA, vitamin B12, folate, erythrocyte sedimentation rate (ESR), and C-reactive protein (CRP). However, these may vary across centers depending on availability and cost [2].

There are plenty of potential alternatives that can act as a mimic for MS. There is no clear guideline for excluding alternative diagnoses, which is usually left to the judgment of the treating physician. Evidence for systemic autoimmune disease with respect to MS is usually searched to exclude possible alternative diagnoses, including systemic lupus erythematosus (SLE) and 
Sjogren's syndrome. Further, examination for antinuclear autoimmune antibody (ANA), an autoantibody group against nuclear and cellular antigens, is a common practice. These antibodies are often indicative of immune dysregulation [3]. However, not all ANA-positive results are clinically meaningful or a cause of concern. These antibodies react with $>150$ nuclear antigens and are not all associated with known autoimmune diseases [4]. ANA is a commonly ordered test with respect to suspected autoimmune diseases, including SLE and other rheumatological diseases. The ANA test is sensitive but nonspecific for SLE diagnosis since ANA can be found in different types of autoimmune diseases, including scleroderma and Sjogren's syndrome, as well as occasionally in healthy individuals [5].

Clinicians frequently order tests for serum autoantibodies, especially ANA, because some rheumatological diseases can present with neurological symptoms resembeling MS. However, ANA testing is generally considered to have a low-positive predictive value [6]. The significance of ANA testing in patients with MS remains unclear. There have been limited studies on the association between MS and ANA with most being performed before the introduction of the 2000 McDonald criteria for MS diagnosis. A previous study was conducted before 2000 to examine whether patients with MS who were ANA positive should be excluded from clinical trials and reported no difference between patients with MS with low ANA titer and those that were ANA negative [7]. A recent study tried to look at the prevalence of positive autoantibodies in patient with MS in Vienna and reported $18 \%$ of the patients with MS had positive autoantibody without having another autoimmune disease [8]. However, these findings could not be generalized in different cohorts with different ethnic characteristics and prevalence of MS and other autoimmune diseases.

The specific ANA value in MS diagnosis remains unclear; however, an increased level should be indicative of an alternative diagnosis. Previous studies have reported an incidence of positive ANA in patients with MS ranging from 2 to 44\% [9]. The correlation between ANA titer and the disability extent remains unclear. This study aimed to compare the prevalence of positive ANA antibody and its titer between patients with MS and patients with general neurological diseases in Saudi Arabia. Further, we aimed to examine the association between the ANA level and disease disability by measuring the Expanded Disability Status Score (EDSS).

\section{Methods}

In this case-control study, we reviewed the ANA results for all patients who attended the neurology MS clinic between January 2018 and January 2020. This was a convenient sample that included all MS patients. Data was collected from the health information system (HIS). The inclusion criteria for the cases were as follows: patients with MS diagnosis according to 2017 McDonald criteria. We excluded patients with co-existent rheumatological or autoimmune diseases other than MS according to clinical or laboratory findings. The cases were age and sex matched with the control group. We included a control group of patients with general neurology problems other than MS who attended the neurology clinic at the same period. Inclusion criteria for the control group: Age and sex matched to cases. Not known to have MS or a coexistent diagnosis of autoimmune or rheumatological diseases. Patients who did not have a final diagnosis or have a neurological disease of unknown cause were excluded from the control. Informed written consent was obtained from the participants. The study was approved by Institutional Review Board (IRB) at King Abdulaziz University Hospital.

\section{ANA assay}

All the participants (case and control) underwent the indirect immunofluorescence (IIF ) ANA assay on Human epithelial type 2 (HEp-2)-cell-based IIF at king AbdulAziz University Hospital and visually evaluated by lab technician. ANA titers $\geq 40$ is considered positive. Furthermore, ANA level were categorized to $\leq 160, \leq 640$, and $\geq 1280$ mildly positive, moderately positive, and strongly positive, respectively. All participants with positive ANA were carefully evaluated to exclude any coexistent rheumatological disease. Cases with moderate positive ANA titer or higher were referred to rheumatology service to eliminate any rheumatological causes.

\section{Statistical analysis}

All data analyses were performed using Statistical Package for the Social Sciences (SPSS) software version 23.By ( IBM Corp. Released 2015. Armonk, NY,USA). Descriptive statistics were used as the number of cases, percentages, mean $\pm \mathrm{SD}$ and median (range). The chi-square test for comparison of categorical variables whereas the student $T$ test for comparison of mean and median values respectively. $P$ value was interpreted as statistically significant if it was $<0.05$.

\section{Results}

The total number of participants was 218, and MS cases and control group were 115 and 103, respectively. In MS group, 67 (58.2\%) and 48 (41.7\%) females and males, respectively. Similarly control group had more females than males, $81(78 \%)$ and $22(21.3 \%)$ females and males, respectively. The mean EDSS in the MS group was 1.7. The mean age was 33 and 34 years in the MS and control group, respectively. The disease duration ranged 
from 1 to 30 years, with a mean of 7 years. The majority were relapsing remitting MS 98\%, and $13 \%$ and $1.7 \%$ were secondary progressive MS and primary progressive MS, respectively. Table 1 describes the baseline characteristics. Among the 115 patients with MS, 82\% were on disease-modifying therapy while $18 \%$ were not on treatment. The most frequently used medication was injectable interferon. The control group included patient with no autoimmune diseases 59\% primary headache disorder, $33 \%$ cervical or lower back pain secondary to degenerative disc diseases or muscular spasm, $4 \%$ syncope or dizziness, and $4 \%$ for peripheral vertigo.

Regarding ANA serology in patients with MS, 25.2\% and $74.8 \%$ of the patients with MS were negative and positive, respectively. Among the positive cases, 60\%, $11.3 \%$, and $3.4 \%$ were mildly positive, moderately positive, and strongly positive for ANA, respectively. In the control group, $34.9 \%$ and $65 \%$ of the participants were negative and positive for ANA; among the positive ones, $60 \%$ were mildly positive with none being strongly positive. We performed the chi-squared test for betweengroup comparisons of ANA, which revealed that there were numerically, but not significantly, more positive cases in the MS group (74\%) than in the control group $(65 \%)(p=.117)$.

There was no significant within-group difference in the MS group with respect to the EDSS and ANA titer level (Table 2).

\section{Discussion}

This study aimed to compare the ANA prevalence between a convenience sample of patients with MS and individuals with non-autoimmune neurological diseases. Our findings indicated that positive ANA titer in patients with MS is not rare but did not significantly differ from the control group. Most ANA-positive patients were only positive at the low-range titer. This suggests that routine ANA testing in patients with MS is unnecessary. Further, ANA presence in patients with MS should not prompt the treating physician to perform additional workup with respect to typical clinical and radiological MS findings included in the 2017 McDonald criteria. This is especially when the ANA titer is in the mild to moderate range. Among the patients with MS, there was no difference in the disease severity between ANA-positive and negative patients.

The prevalence of ANA and other autoimmune antibodies in patient with MS have been controversial, probably due to different study design, population characteristic, and the updates in the MS diagnostic criteria over time. The overall incidence of ANA antibody in general population from reported literatures varies between $13 \%$ and $50 \%$ mostly with low titer (1:40). Higher prevalence of ANA were found in female, older age, and certain ethnicity such as African American [5, 10]. Previous studies suggest that ANA testing has a low predictive value if done without clinical suspicion of a rheumatological disease [6]. In patient with MS-positive, ANA reported in around $13 \%$ of the patients $[7,11]$. In a study of 176 individuals with definite MS, testing for autoimmune antibodies were not cost-effective as $18 \%$ of the patients had elevated autoimmune antibodies but minority developed another autoimmune disease over 10 years follow-up [8].

MS diagnosis should be based on the current 2017 McDonald criteria, which requires clinical or paraclinical evidence of dissemination in time and space with the exclusion of any alternative diagnoses [1]. Early diagnosis is essential for preventing long-term disability and improving the prognosis $[12,13]$. ANA has been associated with different autoimmune diseases. Searching for autoantibodies is a crucial step in autoimmune disease workup. However, it is still controversial if MS-positive ANA should be evaluated further or treated differently than ANA-negative MS cases. Some patients who have positive ANA never develop any disease [11]. However, its significance in patients with MS remains unclear [7]. There have been several reports of patients with SLE whose initial clinical presentations were similar to those of MS but were ANA positive [14]. We suggest that this

Table 1 Baseline characteristics of the MS and control groups

\begin{tabular}{|c|c|c|}
\hline Characteristics & Case, $n=115$ & Control, $n=103$ \\
\hline Mean age, years (SD) & $33( \pm 8.9)$ & $34( \pm 8.5)$ \\
\hline \multicolumn{3}{|l|}{ Gender } \\
\hline Male & $48(41.7 \%)$ & $22(21.3 \%)$ \\
\hline Female & 67 (58.2\%) & $81(78.64 \%)$ \\
\hline EDSS & $1.7(\mathrm{SD} \pm 1.9)$ & \\
\hline Disease duration & 7 years $(S D \pm 6.5)$ & \\
\hline Type of MS & 98\% RRMS,13\%SPMS,1.7\%PPMS & \\
\hline ANA positivity & $74.8 \%$ & $65 \%$ \\
\hline
\end{tabular}

. 
Table 2 Relation between ANA titer and mean EDSS

\begin{tabular}{lllll}
\hline ANA titer & Mean EDSS & $\boldsymbol{N}$ & Std. deviation & $\boldsymbol{P}_{\text {value between groups }}$ \\
\hline Negative & 2.0 & 29 & 2.32199 & 0.37 \\
Mild positive & 1.5 & 69 & 1.76418 & 0.83 \\
Moderate positive & 1.7 & 13 & 2.13713 & 0.4 \\
Strong positive & 2.1 & 4 & 2.39357 & 0.93 \\
Total & 1.7 & 115 & 1.96277 &
\end{tabular}

ANA level was categorized into $\leq 160, \leq 640$, and $\geq 1280$ mildly positive, moderately positive, and strongly positive, respectively ANA antinuclear antibody, ${ }^{* *} E D S S$ expanded disability status scale, ${ }^{* * *} N$ number ${ }^{\ddagger}$ ANOVA test was used $P$ value is statistically significant if $\leq 0.05$

inconsistency in findings could be attributed to the fact that these studies were performed before the introduction of the McDonald criteria for MS diagnosis. Therefore, such findings should not be generalized.

\section{Conclusion}

In our study, we focused on the presence of serum ANA in patients with definite MS. We aimed to reassess the values of ANA testing in MS patients. To look if positive ANA can serve as a prognostic marker for disease severity. We concluded that mild ANA positivity in patients with MS is not uncommon and does not differ significantly with that in patients with other neurological disorders. Finding positive ANA in MS patients was not associated with a higher disability score. ANA testing should not be part of routine MS screening for excluding alternative diagnoses unless prompted by a remarkable history or clinical finding.

\section{Study limitation}

This is a retrospective study which has all the restrictive features of the retrospective design. The control group was not healthy patients, which can be better in representing the general population. Although the sample size was comparable to similar studies, conducting a larger sample size and comparing it to the general population could confirm this study.

\section{Abbreviation}

MS: Multiple sclerosis; ANA: Antinuclear antibody; SLE: Systemic lupus erythematosus; ESR: Erythrocyte sedimentation rate; CRP: C-reactive protein; EDSS: Expanded disability status scale; IIF: Indirect immunofluorescence; HEp2: Human epithelial type 2; IRB: Institutional Review Board

\section{Acknowledgements}

We would like to thank Editage [http://www.editage.com] for editing and reviewing this manuscript for English language.

\section{Authors' contributions}

HA has analyzed the data and wrote the results and the draft of the manuscript. FA has reviewed the data and and was a major contributor in writing the manuscript. The authors read and approved the final manuscript.

\section{Funding}

The study was not funded.
Availability of data and materials

The datasets used during the current study are available from the corresponding author on reasonable request.

\section{Ethics approval and consent to participate}

This study was approved by the research ethic community at King Abdelaziz University Hospital on February 3, 2019, reference number (18-19). An informed written consent was obtained from the participants.

Consent for publication

Not applicable.

\section{Competing interests}

The authors declare that they have no competing interests.

\section{Author details}

${ }^{1}$ Neurology Division ,Department of internal Medicine, Faculty of Medicine, King Abdulaziz University, Jeddah, Saudi Arabia. ${ }^{2}$ Imam Abdulrahman Bin Faisal University, Department of Neurology, King Fahad Hospital of the University, Dammam, Saudi Arabia.

Received: 29 July 2020 Accepted: 1 February 2021

Published online: 15 February 2021

\section{References}

1. Aktas $O$, Wattjes MP, Stangel M, Hartung HP. Diagnosis of multiple sclerosis: revision of the McDonald criteria 2017. Nervenarzt. $2018 ; 89(12)$.

2. Calabrese M, Gasperini C, Tortorella C, Schiavi G, Frisullo G, Ragonese P, et al. "Better explanations" in multiple sclerosis diagnostic workup: a 3-year longitudinal study. Neurology. 2019;92(22).

3. Fatemi G, Kermani TA. Clinical significance of a positive antinuclear antibody test. JAMA.2015;314(8).

4. Bernardini S, Infantino M, Bellincampi L, Nuccetelli M, Afeltra A, Lori R, et al. Screening of antinuclear antibodies: comparison between enzyme immunoassay based on nuclear homogenates, purified or recombinant antigens and immunofluorescence assay. Clin Chem Lab Med. 2004;42(10).

5. Marin GG, Cardiel MH, Cornejo H, Viveros ME. Prevalence of antinuclear antibodies in 3 groups of healthy individuals: blood donors, hospital personnel, and relatives of patients with autoimmune diseases. J Clin Rheumatol. 2009;15(7)

6. Abeles AM, Abeles M. The clinical utility of a positive antinuclear antibody. AJM. 2013;126(4).

7. Tourbah A, Clapin A, Gout O, Fontaine B, Liblau R, Batteux F, et al. Systemic autoimmune features and multiple sclerosis: a 5-year follow-up study. Arch Neurol 1998:55(4).

8. Dal-Bianco A, Wenhoda F, Rommer PS, Weber M, Altmann P, Kraus J, et al. Do elevated autoantibodies in patients with multiple sclerosis matter? Acta Neurol Scand. 2019;139(3).

9. Series C. A challenging entity: multiple sclerosis or collagen tissue disorders: a case series of 6 patients. Caspian J Intern Med. 2017;8(4).

10. Satoh M, Chan EKL, Ho LA, Rose KM, Parks CG, Cohn RD, et al. Prevalence and sociodemographic correlates of antinuclear antibodies in the United States. Arthritis Rheum 2012;64(7).

11. Lang K, Prüss H. Frequencies of neuronal autoantibodies in healthy controls. Neurol - Neuroimmunol Neuroinflammation. 2017;4(5).

12. Van der Vuurst de Vries RM, Mescheriakova JY, Wong YYM, Runia TF, Jafari N, Samijn JP, et al. Application of the 2017 revised mcdonald criteria for 
multiple sclerosis to patients with a typical clinically isolated syndrome. JAMA Neurol. 2018 1;75(11)

13. Tintore M, Rovira À, Río J, Otero-Romero S, Arrambide G, Tur C, et al.

Defining high, medium and low impact prognostic factors for developing multiple sclerosis. Brain. 2015;138(7).

14. van der Kaaden AJ, Kamphuis DJ, Nossent JC, Rico RE. Longstanding

isolated cerebral systemic lupus erythematosus in an 8-year-old black girl. Resemblance with multiple sclerosis. Clin Neurol Neurosurg. 1993;95(3).

\section{Publisher's Note}

Springer Nature remains neutral with regard to jurisdictional claims in published maps and institutional affiliations.

Submit your manuscript to a SpringerOpen ${ }^{\circ}$ journal and benefit from:

- Convenient online submission

Rigorous peer review

- Open access: articles freely available online

High visibility within the field

- Retaining the copyright to your article

Submit your next manuscript at $\boldsymbol{\sim}$ springeropen.com 